\title{
Pathologically Proven Gadolinium-enhanced MRI Lesions in the Bilateral Corticospinal Tracts in Lymphomatosis Cerebri
}

\author{
Genpei Yamaura ${ }^{1}$, Akihiro Ogasawara ${ }^{1}$, Takeshi Ito $^{1}$, Shizuka Ohsugi ${ }^{1}$, Yoichi Kanatsuka ${ }^{1}$, \\ Ryuichiro Hayashi ${ }^{1}$, Hiromichi Iwashita ${ }^{2}$, Hiroyuki Hayashi ${ }^{2}$, Shigeru Koyano ${ }^{3,4}$, \\ Shigeki Yamaguchi ${ }^{1}$ and Fumiaki Tanaka ${ }^{4}$
}

\begin{abstract}
:
A 78-year-old woman in complete remission of mass-forming primary central nervous system lymphoma (PCNSL) showed diffuse leukoencephalopathy as well as corticospinal tract lesions with intense gadolinium enhancement on magnetic resonance imaging (MRI). She died 3 months later. In line with the MRI findings, pathological examination revealed dense infiltration of atypical lymphoid cells, consistent with a diagnosis of lymphomatosis cerebri (LC)-type PCNSL. This is the first report of LC in which the corticospinal tracts demonstrated robust contrast enhancement directly corresponding to the neuropathological findings, and it is also a rare instance in which LC presented as a recurrence of typical PCNSL.
\end{abstract}

Key words: corticospinal tracts, diffuse large B-cell lymphoma, gadolinium-enhanced lesions, lymphomatosis cerebri, pathological findings, recurrence

(Intern Med 59: 2931-2934, 2020)

(DOI: 10.2169/internalmedicine.4382-19)

\section{Introduction}

Primary central nervous system lymphoma (PCNSL) usually presents as a hyperintense, homogeneous, gadoliniumenhanced solitary mass lesion on magnetic resonance imaging (MRI) (1). On the other hand, lymphomatosis cerebri (LC), a rare variant of PCNSL, is radiologically characterized by diffuse and asymmetric leukoencephalopathy without contrast enhancement (2). It is therefore rare for typical PCNSL to recur as LC-type PCNSL (3).

We herein describe the case of a 78-year-old female patient with LC who presented with hyperintense gadoliniumenhancing MRI lesions in the bilateral corticospinal tracts 5 years after achieving remission of mass-forming typical PCNSL. A post-mortem histological examination revealed a dense infiltration of lymphoid cells in the corticospinal tracts, which corresponded well to the robust contrast enhancement on MRI.

\section{Case Report}

A 72-year-old woman developed gait disturbance and was admitted to a nearby hospital. A fluid-attenuated inversion recovery (FLAIR) MRI showed a hyperintense mass lesion in the right temporal lobe, with partial gadolinium enhancement on T1-weighted imaging (Fig. 1a, b). Following a stereotactic brain biopsy, the patient was pathologically diagnosed with PCNSL of the diffuse large B-cell lymphoma (DLBCL) type. She received multi-agent chemotherapy, including pirarubicin, cyclophosphamide, vincristine, methotrexate, and prednisone, and achieved a first complete remission. At 73 years of age, her PCNSL recurred in the right frontal lobe without any accompanying neurological deficits, and she received high-dose methotrexate (HDMTX), resulting in a second complete remission.

Five years later, at 78 years of age, she was admitted to our hospital because of an acute disturbance of conscious-

\footnotetext{
${ }^{1}$ Department of Neurology, Yokohama Municipal Citizen's Hospital, Japan, ${ }^{2}$ Department of Pathology, Yokohama Municipal Citizen's Hospital, Japan, ${ }^{3}$ Department of Neurology, Yokohama Minami Kyousai Hospital, Japan and ${ }^{4}$ Department of Neurology and Stroke Medicine, Yokohama City University Graduate School of Medicine, Japan

Received: December 26, 2019; Accepted: June 4, 2020; Advance Publication by J-STAGE: July 21, 2020

Correspondence to Dr. Tanaka Fumiaki, ftanaka@yokohama-cu.ac.jp
} 

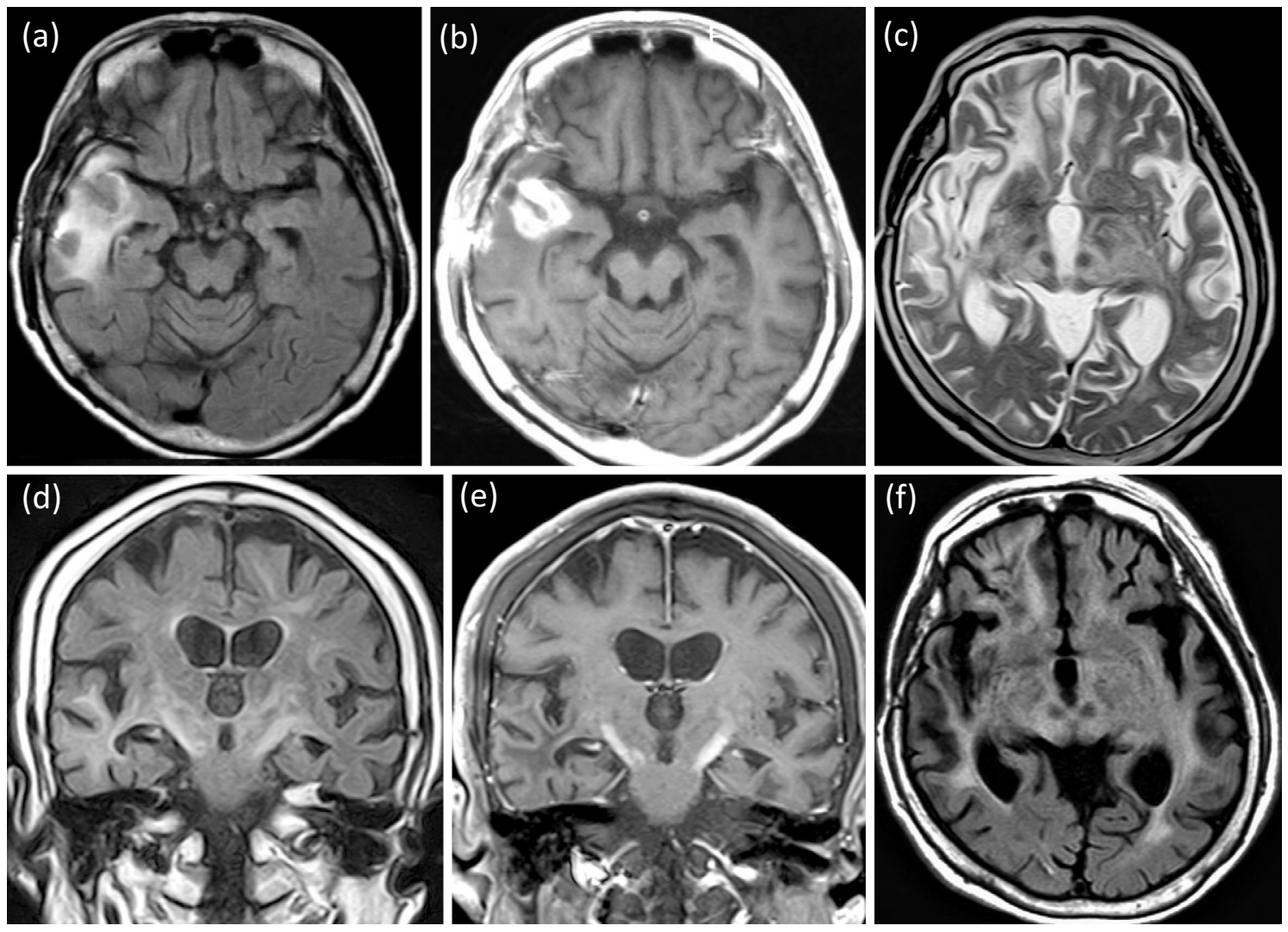

Figure 1. Findings on magnetic resonance imaging. (a, b) 73 years, (c, d, e) 78 years, (f) 1 month prior to death. (a) Hyperintense lesion in the right temporal lobe on a FLAIR image. (b) Gadoliniumenhancing mass lesion in the right temporal lobe on a T1-weighted image. (c) Diffuse hyperintensities in the left frontal lobe and bilateral diencephalon, and old lesions in the right temporal and frontal lobes on a T2-weighted image. (d) Hyperintense signals in the bilateral corticospinal tracts on a coronal FLAIR image. (e) Gadolinium-enhancing bilateral corticospinal lesions on a coronal T1-weighted image. (f) Diffuse hyperintensities in the bilateral cerebral hemispheres, sparing the left occipital lobe, on a FLAIR image. FLAIR: fluid-attenuated inversion recovery

ness [Glasgow Coma Scale score of 13 (E3V4M6)]. Here neurological findings were otherwise normal, including the absence of pyramidal signs. The results of hematological examinations, including a complete blood count and blood chemistry, were normal. A cerebrospinal fluid examination showed a slight elevation of the cell count and protein level $(6 / \mu \mathrm{L}$ and $66 \mathrm{mg} / \mathrm{dL}$, respectively), but cytology yielded negative results for malignant cells.

T2-weighted MRI of the brain revealed newly developed diffuse abnormal hyperintensities in the left frontal lobe and bilateral diencephalon, and old lesions in the right temporal and frontal lobes (Fig. 1c). Hyperintense lesions were also observed in the bilateral corticospinal tracts on coronal FLAIR MRI (Fig. 1d). Notably, on a T1-weighted coronal image only the corticospinal lesions showed symmetric, dense gadolinium enhancement (Fig. 1e). Either a rerecurrence of PCNSL or a new neuroinflammatory disorder was suspected, and the patient was emergently treated with high-dose intravenous methylprednisolone. However, her condition rapidly deteriorated and she died 3 months after admission.

A postmortem examination showed that atypical lymphoid cells with large and irregular-shaped nuclei had densely infiltrated the corticospinal tracts (Fig. 2a), consistent with the robust contrast enhancement observed on MRI. On an immunohistological examination, these lymphoid cells were strongly positive for CD20, a pan-B cell marker (Fig. 2b). A few reactive $\mathrm{T}$ cells positive for $\mathrm{CD} 3$ were observed in the vicinity of neoplastic B cells (Fig. 2c). Scattered, atypical lymphoid cells were also observed in the left occipital lobe around the calcarine sulcus (Fig. 2d), where no abnormalities were found on MRI performed 1 month before death (Fig. 1f).

No cohesive mass lesions with malignant lymphoid cells were observed in the brain, including the corticospinal tracts, resulting in a diagnosis of a re-recurrence of highgrade DLBCL of the LC type.

\section{Discussion}

LC is a rare variant of PCNSL that is characterized by the diffuse infiltration of malignant lymphoma cells without formation of a discernible cohesive tumor mass. Originally, the MRI features of LC were reported to consist of diffuse leu- 

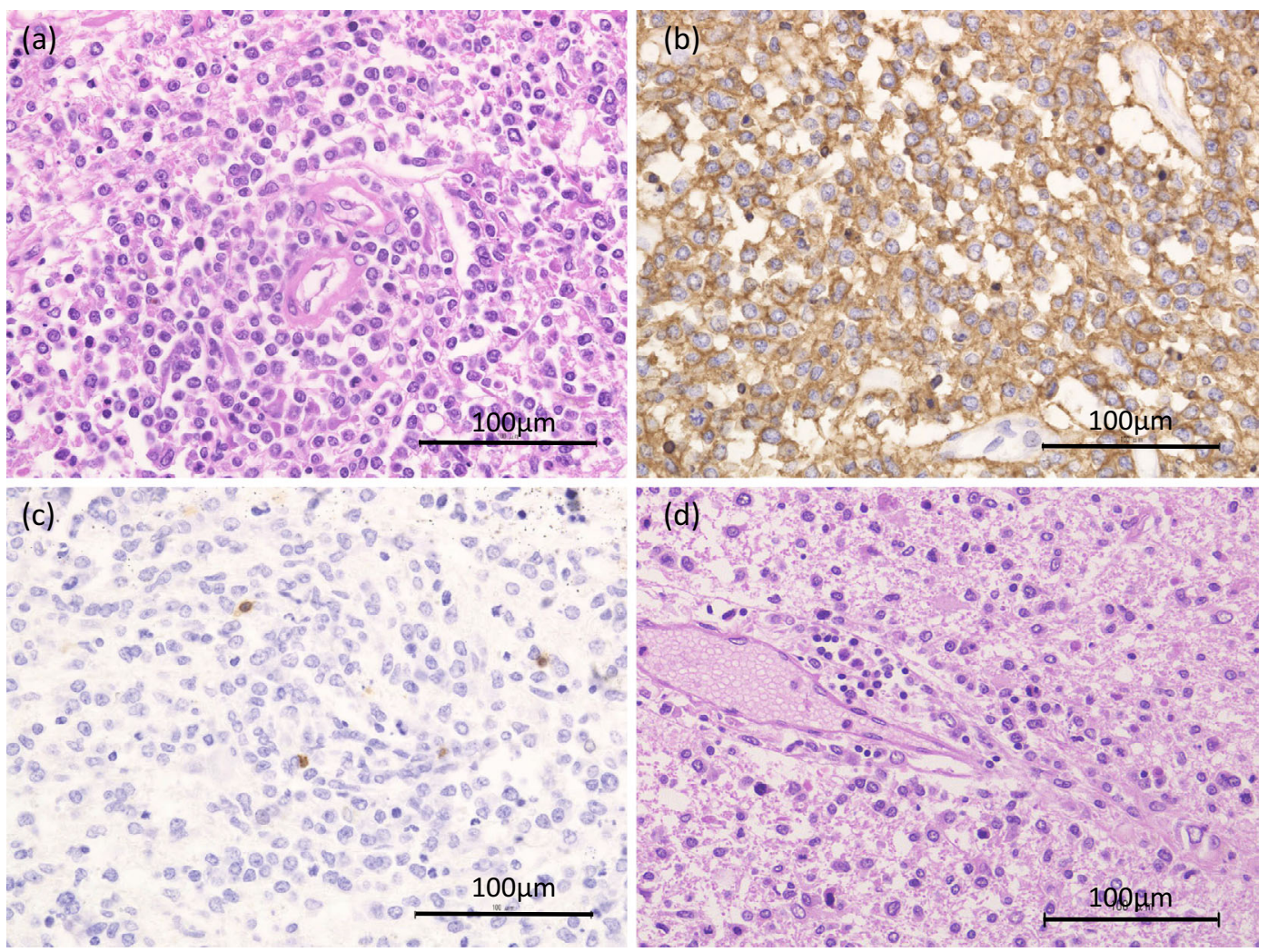

Figure 2. Pathological findings. (a) Atypical lymphoid cells with large and irregularly shaped nuclei are densely infiltrating the corticospinal tracts [Hematoxylin and Eosin (H\&E) staining]. (b) Neoplastic lymphoid cells are positive for $\mathrm{CD20}$. (c) A few reactive $\mathrm{T}$ cells positive for $\mathrm{CD3}$ are observed around neoplastic B cells. (d) Scattered neoplastic lymphoid cells are observed in the left occipital lobe around the calcarine sulcus (H\&E staining).

koencephalopathy in the cerebral hemispheres with no contrast enhancement, reflecting an intact blood-brain barrier (BBB) (2). However, subtle, patchy, or even mass-like enhanced lesions have been reported in the later stages of LC (4-7). A recent case series characterized the neuroradiological findings of LC and revealed that a relatively high percentage of patients $(35.6 \%)$ demonstrated spreading of lymphoid cells along the corticospinal tracts (4). The patient in our case presented with an impressively robust enhancement of the bilateral corticospinal tracts following the administration of a gadolinium contrast agent. No previous reports have shown such intense contrast enhancement of the corticospinal tracts in LC, and moreover, such lesions have never been confirmed neuropathologically. Based on our pathological finding of a dense infiltration of lymphoid cells in the corticospinal tracts (Fig. 2a), we assume that the BBB might have been disrupted by the aggressive invasion of these cells, and that this disruption corresponded to the robust contrast enhancement on MRI. This differs from the non-contrast-enhancing lesions in typical LC, where diffusely infiltrative lymphoid cells do not affect the structure of the BBB (4).

It is also interesting to note that our patient was initially diagnosed with typical mass-forming PCNSL, and she achieved a complete remission, then 5 years later developed LC, an atypical, diffuse form of PCNSL. Only one previous case report pathologically confirmed that typical PCNSL recurred as LC-type PCNSL (3). However, a recent imaging study showed that atypical, non-enhanced T2-weighted/ FLAIR hyperintense lesions, possibly indicating LC, coexisted with typical enhancing PCNSL lesions (8). The former lesions could constitute distinct sites of relapse (8). Although the authors of this past study did not confirm the pathology of these lesions (8), the recurrence of typical PCNSL as LC-type PCNSL might not be as rare as previously considered. In the radiological diagnosis of PCNSL, attention should be paid to possible changes in the histological type as the disease progresses or recurs, because the radiological findings are quite different between mass-forming PCNSL (Fig. 1a, b) and LC (Fig. 1c-f).

Although our patient's mass-forming PCNSL recurred only 1 year after intensive chemotherapy that included methotrexate, the second remission, which was achieved after treatment with HD-MTX, lasted for 5 years. In two retrospective studies $(9,10)$ of relapsed PCNSL, re-treatment with HD-MTX resulted in median progression-free survival durations of 16 and 25.8 months, respectively. Furthermore, in one of these studies, the degree of functional impairment was a prognostic factor for the progression-free survival (9). The long-term effectiveness of HD-MTX treatment during the second remission in our case might be attributed to the lack of neurological deficits at relapse. On the other hand, 
the prognosis of LC-type PCNSL is extremely poor, and the median overall survival was reported to be only 2.95 months (11), consistent with the 3-month survival after rerecurrence as LC in our case. Although our patient did not receive methotrexate for $\mathrm{LC}$, its administration has been reported to prolong the median overall survival of LC patients by up to 13.8 months (11).

In conclusion, our patient with mass-forming PCNSL demonstrated an unusual recurrence in the form of LC, with intensely enhanced lesions in the bilateral corticospinal tracts which has not been previously described. Moreover, the densely infiltrative lymphoid cells were pathologically confirmed in the corticospinal tracts.

The authors state that they have no Conflict of Interest (COI).

Shigeru Koyano and Shigeki Yamaguchi contributed equally to this work.

\section{References}

1. Citterio G, Reni M, Gatta G, Ferreri AJM. Primary central nervous system lymphoma. Crit Rev Oncol Hematol 113: 97-110, 2017.

2. Terae S, Ogata A. Nonenhancing primary central nervous system lymphoma. Neuroradiology 38: 34-37, 1996.

3. Matsumoto K, Kohmura E, Fujita T, Tsuruzono K, Tsujimura T, Kawano K. Recurrent primary central nervous system lymphoma mimicking neurodegenerative disease. Neurol Med Chir (Tokyo) 35: 360-363, 1995.
4. Li L, Rong J, Feng J. Neuroradiological features of lymphomatosis cerebri: a systematic review of the English literature with a new case report. Oncol Lett 16: 1463-1474, 2018.

5. Samani A, Davagnanam I, Cockerell OC, Ramsay A, Patani R, Chataway J. Lymphomatosis cerebri. A treatable cause of rapidly progressive dementia. J Neurol Neurosurg Psychiatry 86: 238-240, 2015.

6. Yamamoto T, Kojima K, Koibuchi K, et al. A case of primary central nervous system lymphoma presenting diffuse infiltrative leukoencephalopathy. Intern Med 51: 1103-1106, 2012.

7. Hashiguchi S, Momoo T, Murohashi Y, et al. Interleukin 10 level in the cerebrospinal fluid as a possible biomarker for lymphomatosis cerebri. Intern Med 54: 1547-1552, 2015.

8. Tabouret E, Houillier C, Martin-Duverneuil N, et al. Patterns of response and relapse in primary CNS lymphomas after first-line chemotherapy: imaging analysis of the ANOCEF-GOELAMS prospective randomized trial. Neuro Oncol 19: 422-429, 2017.

9. Pentsova E, DeAngelis LM, Omuro A. Methotrexate re-challenge for recurrent primary central nervous system lymphoma. J Neurooncol 117: 161-165, 2014.

10. Plotkin SR, Betensky RA, Hochberg FH, et al. Treatment of relapsed central nervous system lymphoma with high-dose methotrexate. Clin Cancer Res 10: 5643-5646, 2004.

11. Izquierdo $\mathrm{C}$, Velasco $\mathrm{R}$, Vidal $\mathrm{N}$, et al. Lymphomatosis cerebri: a rare form of primary central nervous system lymphoma. Analysis of 7 cases and systematic review of the literature. Neuro Oncol 18: $707-715,2016$

The Internal Medicine is an Open Access journal distributed under the Creative Commons Attribution-NonCommercial-NoDerivatives 4.0 International License. To view the details of this license, please visit (https://creativecommons.org/licenses/ by-nc-nd/4.0/).

(C) 2020 The Japanese Society of Internal Medicine Intern Med 59: 2931-2934, 2020 